\title{
Eine Option für die Psychoeduktion
}

Fragestellung: Untersuchung zur Wirksamkeit des internetbasierten Selbsthilfeprogramms „Deprexis“ als antidepressive Therapie.

Hintergrund: Nur knapp $60 \%$ aller an einer unipolaren Depression Erkrankten erhalten eine antidepressive Therapie. Um diese Behandlungslücke zu schließen, wird die Wirksamkeit verschiedener internetbasierter Selbsthilfeprogramme untersucht, die als Überbrückungs-, Komplementär- oder Alternativbehandlung bei psychiatrischen Erkrankungen [1] vermehrt genutzt werden.

Patienten und Methodik: Die Rekrutierung der Studienteilnehmer erfolgte mittels Internetplattform, die Diagnosestellung anhand der Selbstaussage der Probanden mit externer Diagnosebestätigung in 29\% der Fälle. Jeweils 105 Probanden wurden in einen Therapie- und einen Wartelistenarm randomisiert. Es standen zehn auf kognitiver Verhaltenstherapie basierende Therapiemodule zur Verfügung. Primärer Endpunkt war die Veränderung im BDI (Beck depression inventory). Die Studiendauer betrug acht Wochen.

Moritz S, Schilling L, Hauschildt $\mathrm{M}$ et al. A randomized controlled trial of internet-based therapy in depression. Behav Res Ther 2012; 50: 513-21
Ergebnisse: Es fand sich eine niedrige Abbruchrate (18\%) mit signifikanten Symptomverbesserungen in beiden Gruppen im Verlauf $(\mathrm{d}=0,85$ im Therapiearm, $\mathrm{d}=0,58 \mathrm{im}$ Wartelistenarm) mit leichter

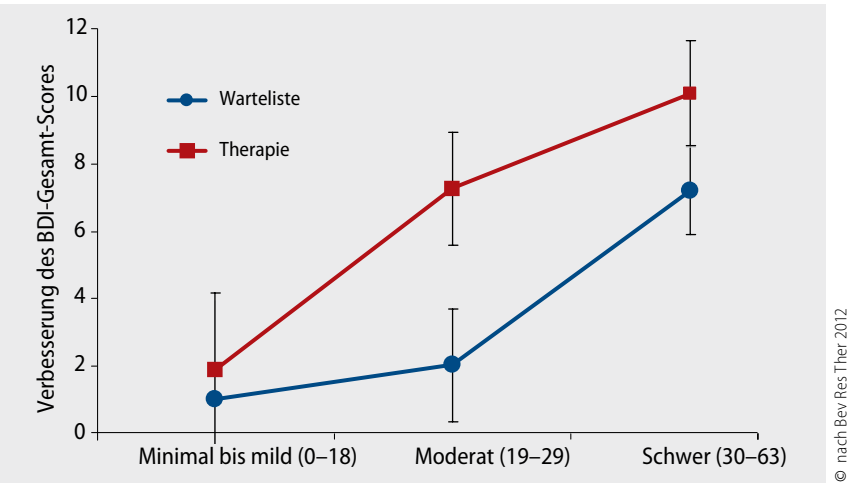

1 Die internetbasierte Intervention erwies sich in diesem Setting als wirksame Therapieoption.

Überlegenheit des Therapiearms $(\mathrm{d}=0,38)$ ( Abb. 1). Der stärkste Effekt wurde in der Gruppe der mittelschweren Depressionen erzielt $(\mathrm{d}=0,70)$, schwache Effekte hingegen bei schweren $(d=0,23)$ und leichten $(d=0,24)$ Fällen. Das Programm wurde von den Teilnehmern retrospektiv generell als wirksam und gut durchführbar beurteilt.

Schlussfolgerungen: Die Ergebnisse deuten auf einen möglichen Einsatz des gut anwendbaren und vom Probanden akzeptierten Programms bei depressiven Syndromen hin. Sie stützen Vorbefunde mit diesem Programm und entsprechen Ergebnissen aus Metaanalysen.

\section{- Kommentar von Frank M. Schmidt und PD Dr. Peter Schönknecht}

\section{Internetbasierte Selbsthilfeprogramme verstärkt nutzen}

In der Befundinterpretation dieser methodisch aufwendigen und praxisrelevanten randomisierten Studie muss eingeschränkt werden, dass der Schweregradeinteilung und der Messung des Behandlungseffekts ausschließlich Selbstbeurteilungsskalen (BDI als primary outcome) zugrunde liegen, die mit fachpsychiatrischer Fremdbeurteilung (z. B. HAMD, MADRS) nicht sicher deckungsgleich sind [2]. Die Rekrutierung mittels Werbung auf Depressions-Internetforen stellt eine Recruitment-Selection dar (in dieser Studie mehr als 77\% Frauen). Die angegebenen Diagnosen beruhen nur zu $60 \%$ auf fachtherapeutischer Expertise, was zu Fehldiagnosen geführt haben kann.

Auf Grundlage der vorliegenden Daten stellt diese Therapieform zumindest für Patienten, die keiner anerkannten Therapieform zugeführt werden können, eine Alternative dar. Es ist ebenso denkbar, dass derartige Therapieformen die Behandlungsbereitschaft für psychiatrische Therapien unterstützen und somit als psychoedukative Interventionen weiterentwickelt werden sollten.

\author{
Referenzen \\ 1. Kirkby KC et al. Stud Health Technol Inform 2010; 154: $73-6$ \\ 2. Schneibel R et al. Psychiatry Res 2012 Mar 21 [Epub ahead of print] \\ 3. Bschor T et al. Acta Psychiatr Scand 2010; 121: 174-9
}

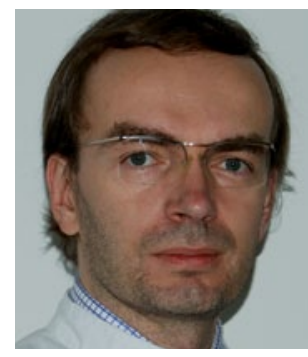

PD Dr. med. Peter Schönknecht, Leipzig

Stellvertretender Klinikdirektor für Forschung und Personal, Klinik und Poliklinik für Psychiatrie und Psychotherapie, Universitätsklinikum Leipzig. E-Mail: peter. schoenknecht@medizin.uni-leipzig.de 